\title{
Review Article \\ Serologic Intestinal-Fatty Acid Binding Protein in Necrotizing Enterocolitis Diagnosis: A Meta-Analysis
}

\author{
Shupeng Cheng, Jialin Yu, Min Zhou, Yan Tu, and Qi Lu \\ Department of Neonatology, Children's Hospital of Chongqing Medical University, Ministry of Education Key Laboratory of \\ Child Development and Disorders, Chongqing 400014, China
}

Correspondence should be addressed to Qi Lu; qilu_qi@hotmail.com

Received 3 October 2015; Accepted 25 November 2015

Academic Editor: Kosmas Paraskevas

Copyright (C) 2015 Shupeng Cheng et al. This is an open access article distributed under the Creative Commons Attribution License, which permits unrestricted use, distribution, and reproduction in any medium, provided the original work is properly cited.

Background. Previous studies showed that intestinal-fatty acid binding protein (I-FABP) may be a valid and promising serologic biomarker for early diagnosis of necrotizing enterocolitis (NEC). Objective. To investigate the early diagnostic value of serologic I-FABP in NEC for the premature neonates. Methods. All major databases were searched from January 1, 1990, to May 1, 2015. We used Meta-Disc 1.4 and Revman5.0 software to calculate the diagnostic accuracy. Results. Seven studies with 444 subjects were identified. The pooled sensitivity of I-FABP was 0.67 for NEC I, 0.74 for NEC II, and 0.83 for NEC III, and the pooled specificity was 0.84 , respectively, which showed a moderate diagnostic accuracy. The area under curve (AUC) for each stage was $0.75\left(Q^{*}=0.69\right)$, $0.82\left(Q^{*}=0.76\right)$, and $0.91\left(Q^{*}=0.84\right)$. The diagnostic threshold analysis showed no significant difference in threshold effect. The metaregression showed that the cut-off value has the largest effect on heterogeneity. The funnel plots indicated the existence of publication bias. Conclusion. I-FABP is a valid serologic biomarker for early diagnosis in NEC for the premature neonates with a moderate accuracy.

\section{Introduction}

Necrotizing enterocolitis (NEC) is a common and devastating condition in neonates and remains a leading cause of morbidity and mortality (20-40\%), especially for the preterm infants [1].

Diagnosis of NEC was made by clinical and radiological signs according to modified Bell's staging criteria at present [2]. However, relying on clinical manifestations tends to heighten omission diagnostic rate because both systemic and abdominal signs are nonspecific in NICU patients. These signs include feeding intolerance, abdominal distention, bloody stool, dyspepsia, and ascites [3]. Similarly, the radiological signs often lack sufficient discriminative power due to time delay [4]. Accurate and timely diagnosis will limit morbidity, improve patients' living quality, and reduce costs. Therefore, we urgently need a new diagnostic method that is valid and promising for early diagnosis.

Serologic intestinal-fatty acid binding protein (I-FABP), a small $(14-15 \mathrm{kDa})$ water-soluble protein, has been studied as an early diagnostic biomarker for NEC in the past decade.
This small cytosolic protein, located mainly in enterocytes of the small intestine, is released into the blood stream after intestinal ischemia and cell disruption [5]. Several studies have suggested the level of I-FABP could be used as a promising biomarker for early diagnosis and the prediction of severe NEC, even possibly for timing of surgery $[6,7]$. Currently, serologic I-FABP has been proved to be available in some medical institutions, but the diagnostic efficacy still remains controversial. For example, multicenter studies with large sample size and systematic review on I-FABP for early diagnosis are lacking. So, we conducted this meta-analysis to systematically evaluate the validity of I-FABP for early diagnosis in NEC.

\section{Methods}

2.1. Data Source. Web of Science, Embase, Medline databases, Cochrane Library, CNKI, VIP, and other Chinese medical databases were searched from January 1, 1990, to May 1, 2015. The search terms were "necrotizing enterocolitis", "NEC", "newborn", "neonate", "biomarker", "I-FABP", and 
"intestinal-fatty acid binding protein". The reference lists from original articles were also examined, and we contacted authors to obtain further information by email, if necessary.

2.2. Inclusion and Exclusion Criteria. Studies that met the following eligibility criteria were included: (1) studies that assessed the diagnostic accuracy of serologic I-FABP in NEC; (2) studies that included case group (neonates who were evaluated with suspected NEC of Bell stage $\geq I$ ) $[2,3]$ and control group (gestational age and weight-matched neonates who were admitted to the same institution without NEC, sepsis, or systemic inflammatory response syndrome); (3) studies that provided sufficient information to construct the two-by-two tables; (4) studies that only contained neonates (within 28 days after birth); (5) studies that had well-defined reference standard and staging criteria for NEC.

Studies were excluded for the following reasons: (1) a letter, case report, and comment and (2) neonates with other gastrointestinal diseases, immunodeficiency disease, inheritance metabolism disorders, and severe congenital malformation. If studies had overlapping subjects, only the most recent information or the largest sample size of patients was included. Articles were independently reviewed by two evaluators, and any disagreements were resolved by consensus.

2.3. Data Extraction and Quality Assessment. Two reviewers independently extracted data from all eligible studies with a predefined information sheet which included country, year of publication, clinical setting, demographics, type of study, sample size, cut-off points, test methods, and true-positive, false-negative, false-positive, and true-negative value. If we needed any additional information that was not reported in the published articles, we requested it through electronic communication with the corresponding authors of the studies. If no reply was received, the studies were excluded from the meta-analysis.

The methodological quality of each included study was assessed based on the quality assessment with diagnostic accuracy (QUADAS) tool including 11 key items. Each item with "yes," "no," and "unclear" answer was scored as 1, -1, and 0 , respectively [8].

2.4. Statistical Analysis. All statistical analyses were carried out using Revman5.0 and Meta-Disc 1.4 software for Windows [9]. We calculated the following measures of each study: sensitivity, specificity, diagnostic odds ratios (DOR), positive likelihood ratio (PLR), and negative likelihood ratio (NLR) with corresponding 95\% confidence intervals, specific to different stages (NEC I, NEC II, and NEC III) [10-13]. To detect heterogeneity, the diagnostic odds ratio (DOR) was graphically displayed using forest plot and analysed using Cochran-Q test $I^{2}$ test. The DOR compares the odds of true-positive patients (=sensitivity) with that of falsepositives ( $=1-$ specificity) and thus summarizes the overall accuracy of a diagnostic test. A $p$ value of less than 0.05 or $I^{2}$ greater than $50 \%$ indicated significant heterogeneity [14]. Fixed-effects model was used if the result of the $Q$ test was not significant; otherwise, the random-effects model was

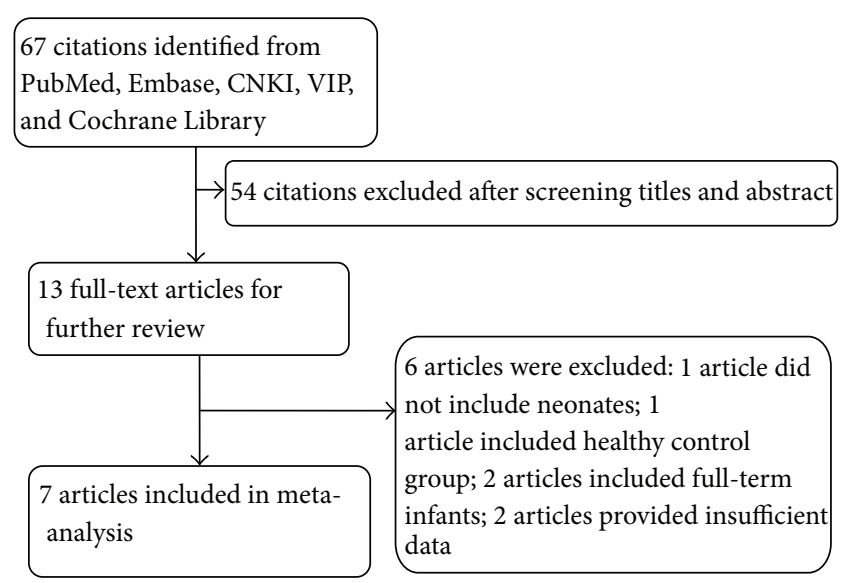

FIgURE 1: Flow diagram highlighting the process of identification and inclusion of studies in the meta-analysis.

used [15]. Then, a summary receiver operator characteristic (SROC) curve was used to summarize these results among all studies and the area under SROC was also calculated to show the diagnostic accuracy. $Q^{*}$ point on the SROC curve was used to obtain the maximum joint sensitivity and specificity [16]. The metaregression analysis was conducted to investigate the confounding factors for heterogeneity, such as cut-off value, study quality (QUADAS), and testing time of plasma samples [17].

Publication bias is common and inevitable in a metaanalysis [18]. As we know, article with a positive result is more likely to be published, following the issue of overestimating the diagnostic performance of serologic I-FABP. In order to solve the problem, we searched different databases for more articles. Besides, publication bias was examined visually by funnel plot, and an asymmetric plot suggested possible publication bias. $p<0.05$ was considered significant.

\section{Results}

3.1. Study Characteristics and Quality Assessment. The literature search was carried out as described, and 13 studies were considered potentially suitable. After full-text review, 4 studies were excluded: one included healthy neonates as controls, two provided insufficient information, and one included patients who were not neonates. In addition, two studies on term neonates were also excluded because of insufficient data. One article with a disagreement between both evaluators was resolved with consensus. Finally, seven publications with 444 neonates met the inclusion criteria and were admitted in the meta-analysis [19-24, 26]. Figure 1 shows the selecting process of studies. The sensitivity, specificity, and true-positive (TP), false-positive (FP), true-negative (TN), and false-negative $(\mathrm{FN})$ value of each article were shown in Figure 2. The cut-off values ranged from $0.76 \mathrm{ng} / \mathrm{mL}$ to $7.70 \mathrm{ng} / \mathrm{mL}$. More detailed characteristics of each included study were presented in Table 1 . All the conditions and methods used for QUADAS of included studies were shown in Figure 3. 


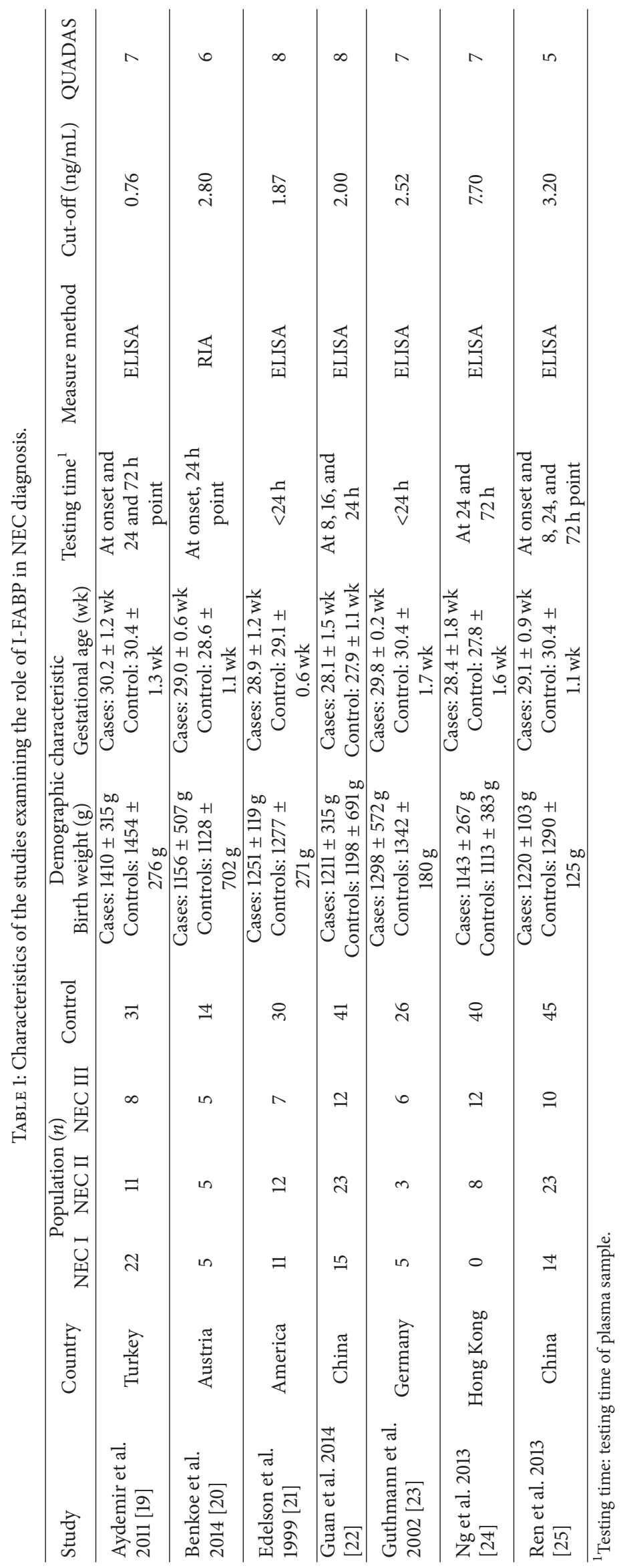




\begin{tabular}{|c|c|c|c|c|c|c|c|c|c|c|c|c|c|}
\hline Study & $\mathrm{TP}$ & FP & FN & $\mathrm{TN}$ & Sensitivity $(95 \% \mathrm{CI})$ & Specificity $(95 \%$ CI) & \multicolumn{3}{|c|}{ Sensitivity $(95 \% \mathrm{CI})$} & \multicolumn{4}{|c|}{ Specificity $(95 \%$ CI) } \\
\hline \multicolumn{14}{|l|}{ NEC I } \\
\hline Aydemir et al. 2011 & 16 & 8 & 6 & 23 & $0.73[0.50,0.89]$ & $0.74[0.55,0.88]$ & & & & & & & $\longrightarrow$ \\
\hline Benkoe et al. 2014 & 3 & 2 & 2 & 12 & $0.60[0.15,0.95]$ & $0.86[0.57,0.98]$ & & & & & & & \\
\hline Edelson et al. 1999 & 8 & 5 & 3 & 25 & $0.73[0.39,0.94]$ & $0.83[0.65,0.94]$ & & & & & & & \\
\hline Guan et al. 2014 & 8 & 8 & 7 & 33 & $0.53[0.27,0.79]$ & $0.80[0.65,0.91]$ & & & & & & & $\longrightarrow$ \\
\hline Guthmann et al. 2002 & 3 & 6 & 2 & 20 & $0.60[0.15,0.95]$ & $0.77[0.56,0.91]$ & & & 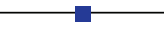 & & & & $\longrightarrow$ \\
\hline \multirow{2}{*}{ Ren et al. 2013} & 10 & 1 & 4 & 44 & $0.71[0.42,0.92]$ & $0.98[0.88,1.00]$ & & & 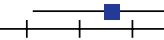 & & & . & $+\quad-$ \\
\hline & & & & & & & 0 & 0.2 & $\begin{array}{lll}0.4 & 0.6 & 0.8\end{array}$ & 1 & 0 & $0.2 \quad 0.4$ & 0.60 .8 \\
\hline \multicolumn{14}{|l|}{ NEC II } \\
\hline Aydemir et al. 2011 & 9 & 8 & 2 & 23 & $0.82[0.48,0.98]$ & $0.74[0.55,0.88]$ & & & - & & & & $\longrightarrow-$ \\
\hline Benkoe et al. 2014 & 4 & 2 & 1 & 12 & $0.80[0.28,0.99]$ & $0.86[0.57,0.98]$ & & & & & & & 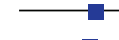 \\
\hline Edelson et al. 1999 & 9 & 5 & 3 & 25 & $0.75[0.43,0.95]$ & $0.83[0.65,0.94]$ & & & 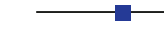 & & & & - \\
\hline Guan et al. 2014 & 13 & 8 & 10 & 33 & $0.57[0.34,0.77]$ & $0.80[0.65,0.91]$ & & & & & & & - \\
\hline Guthmann et al. 2002 & 2 & 6 & 1 & 20 & $0.67[0.09,0.99]$ & $0.77[0.56,0.91]$ & & & & & & & - \\
\hline Ng et al. 2013 & 6 & 7 & 2 & 33 & $0.75[0.35,0.97]$ & $0.82[0.67,0.93]$ & & & 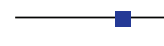 & & & & - \\
\hline \multirow[t]{2}{*}{ Ren et al. 2013} & 20 & 1 & 3 & 44 & $0.87[0.66,0.97]$ & $0.98[0.88,1.00]$ & & & $+\frac{1}{1}$ & & & & \\
\hline & & & & & & & 0 & 0.2 & $\begin{array}{lll}0.4 & 0.6 & 0.8\end{array}$ & 1 & 0 & 0.20 .4 & 0.60 .8 \\
\hline \multicolumn{14}{|l|}{ NEC III } \\
\hline Aydemir et al. 2011 & 6 & 8 & 2 & 23 & $0.75[0.35,0.97]$ & $0.74[0.55,0.88]$ & & & - & & & & $\longrightarrow$ \\
\hline Benkoe et al. 2014 & 5 & 2 & 0 & 12 & $1.00[0.48,1.00]$ & $0.86[0.57,0.98]$ & & & & & & & \\
\hline Edelson et al. 1999 & 6 & 5 & 1 & 25 & $0.86[0.42,1.00]$ & $0.83[0.65,0.94]$ & & & & & & & - \\
\hline Guan et al. 2014 & 8 & 8 & 4 & 33 & $0.67[0.35,0.90]$ & $0.80[0.65,0.91]$ & & & & & & & -6 \\
\hline Guthmann et al. 2002 & 6 & 6 & 0 & 20 & $1.00[0.54,1.00]$ & $0.77[0.56,0.91]$ & & & & $\rightarrow$ & & & - \\
\hline Ng et al. 2013 & 10 & 7 & 2 & 33 & $0.83[0.52,0.98]$ & $0.82[0.67,0.93]$ & & & -6 & & & & $\rightarrow-$ \\
\hline \multirow[t]{2}{*}{ Ren et al. 2013} & 9 & 1 & 1 & 44 & $0.90[0.55,1.00]$ & $0.98[0.88,1.00]$ & & & + & & & +1 & + \\
\hline & & & & & & & 0 & 0.2 & $\begin{array}{lll}0.4 & 0.6 & 0.8\end{array}$ & 1 & 0 & $0.2 \quad 0.4$ & 0.60 .8 \\
\hline
\end{tabular}

FIGURE 2: Forest plot for sensitivity and specificity of I-FABP in NEC diagnosis. TP: true-positive; TN: true-negative; FP: false-positive; FN: false-negative; NEC: necrotizing enterocolitis; I-FABP: intestinal-fatty acid binding protein; CI: confidence interval.

TABLE 2: PLR, NLR, DOR, AUC, and $Q^{*}$ value for each stage.

\begin{tabular}{|c|c|c|c|c|c|c|}
\hline Stage & PLR (95\% CI) & NLR $(95 \%$ CI $)$ & DOR (95\% CI) & $p$ & AUC & $Q^{*}$ \\
\hline NEC I & $3.54(2.29-5.46)$ & $0.45(0.33-0.60)$ & $10.42(2.84-38.28)$ & 0.01 & 0.75 & 0.69 \\
\hline NEC II & $4.23(2.49-7.18)$ & $0.33(0.21-0.51)$ & $15.82(4.37-57.19)$ & 0.00 & 0.82 & 0.76 \\
\hline NEC III & $4.49(2.85-7.09)$ & $0.25(0.15-0.43)$ & $21.26(6.53-69.21)$ & 0.03 & 0.91 & 0.84 \\
\hline
\end{tabular}

PLR: positive likelihood ratio; NLR: negative likelihood ratio; DOR: diagnostic odds ratios; AUC: area under curve; CI: confidence interval; $Q^{*}$ : the maximum joint sensitivity and specificity.

3.2. Diagnostic Accuracy. A random-effects model was used to assess the pooled value of serologic I-FABP in NEC diagnosis because of the potential heterogeneity caused by nonthreshold effect. For each stage, the sensitivity (0.67 [0.55$0.77], 0.74$ [0.63-0.83], and 0.83 [0.71-0.92]), specificity $(0.84$ [0.78-0.89]), and DOR (10.42 [2.84-38.28], 15.82 [4.3757.19], and 21.26 [6.53-69.21]) were performed by forest plots (Figure 2), and the PLR and NLR were showed in Table 2. All the results suggested a moderate accuracy of I-FABP for early diagnosis in NEC.

3.3. Analysis of Heterogeneity. Heterogeneity can signally influence the diagnostic accuracy of a meta-analysis. In this meta-analysis, heterogeneity that was explored was cutoff values, study quality (QUADAS), and testing time of plasma samples. First, we explored the threshold effect with calculating the Spearman correlation coefficient with Moses' model weighted by inverse variance. The results showed no threshold effect $\left(p_{\mathrm{I}}=0.53, p_{\mathrm{II}}=0.48\right.$, and $\left.p_{\mathrm{III}}=0.61\right)$. Then, we used the forest plot of diagnostic odds ratios to assess the nonthreshold effect with random-effects model, and Cochran- $Q$ value suggested the nonthreshold effect was statistically significant for each stage $\left(p_{\mathrm{I}}=0.01, p_{\mathrm{II}}=0.00\right.$, and $\left.p_{\text {III }}=0.03\right)$ (Figure 4, Table 2).

The reasons for heterogeneity were explored by metaregression analysis with Meta-Disc 1.4 software. The process was turning the variations such as cut-off value, study quality (QUADAS), and testing time of plasma samples from left "covariates" to the right "model" and then removes the covariate and analyzes accordingly the descending $p$ values, respectively. The results showed that cut-off value was the main factor for heterogeneity $(\mathrm{RDOR}=4.41, p$ value $=$ $0.0650)$ in Table 3. 


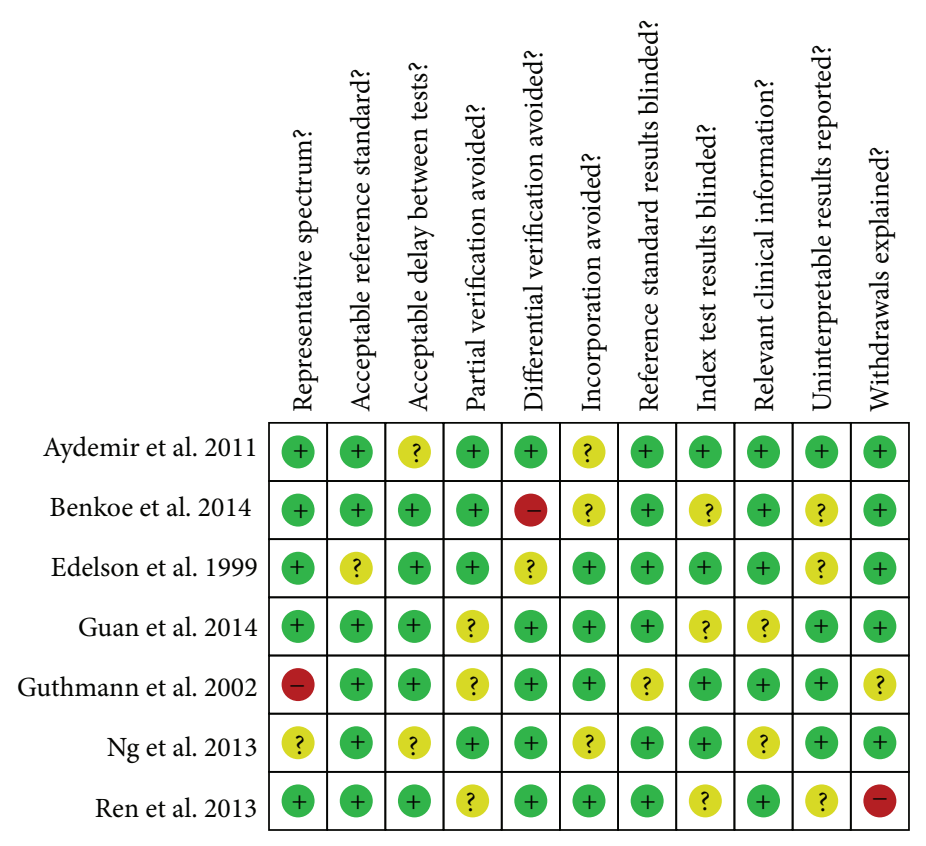

FIGURE 3: QUADAS results about the level of risk of bias for included studies.

3.4. Analysis of SROC. We found that the summary receiver operating characteristic (SROC) curve was positioned near the upper left corner of the curve. The maximum joint sensitivity and specificity $\left(Q^{*}\right.$ value) was 0.69 for NEC I, 0.76 for NEC II, and 0.84 for NEC III, and the area under curve (AUC) was $0.75,0.82$, and 0.91 for each stage, consistent with a moderate diagnostic accuracy of I-FABP for early diagnosis in NEC (Figure 5, Table 2).

3.5. Publication Bias. The visual funnel plot was asymmetric, showing a potential publication bias among studies. Considering the similarity between the three funnel plots for each stage, we just selected one at random (Figure 6).

\section{Discussion}

Necrotizing enterocolitis is one of the most severe diseases and an important cause of mortality and morbidity in neonates. Early symptoms and clinical signs are nonspecific, and the radiological signs often lack sufficient discriminative power. Therefore, it is necessary for us to study valid markers for future research of NEC.

In this meta-analysis, we can find that the area under curve (AUC) was $0.75,0.82$, and 0.91 and $Q^{*}$ value in SROC curve was $0.69,0.76$, and 0.84 for each stage, indicating a moderate pooled accuracy of I-FABP in diagnosing NEC. Similarly, the pooled values of sensitivity (0.67 [0.55-0.77], $0.74[0.63-0.83]$, and $0.83[0.71-0.92])$ and specificity $(0.84$ [0.78-0.89]) suggested the potential diagnostic value of IFABP for early detection, and DOR (10.42 versus 15.82 versus 21.26) also showed a moderate diagnostic accuracy for diagnosing NEC.
TABLE 3: Metaregression analysis of the effects of some covariates on I-FABP in diagnosis of NEC.

(a)

\begin{tabular}{lcccc}
\hline Covariates & Coefficient & Stand. error & RDOR $(95 \%$ CI $)$ & $p$ value \\
\hline Cut-off & 0.508 & 0.3570 & $4.41(1.25 ; 16.47)$ & 0.0650 \\
QUADAS & 0.899 & 0.9412 & $1.53(0.19 ; 11.82)$ & 0.7290 \\
Testing time & 1.319 & 1.3255 & $2.36(0.69 ; 21.44)$ & 0.2862 \\
\hline
\end{tabular}

(b)

\begin{tabular}{lcccc}
\hline Covariates & Coefficient & Stand. error & RDOR $(95 \% \mathrm{CI})$ & $p$ value \\
\hline Cut-off & 0.659 & 0.5387 & $5.16(1.87 ; 14.11)$ & 0.1847 \\
Testing time & 1.587 & 1.8118 & $2.35(0.58 ; 23.35)$ & 0.4376 \\
\hline
\end{tabular}

(c)

\begin{tabular}{llll}
\hline Covariates Coefficient & Stand. error & RDOR $(95 \% \mathrm{CI})$ & $p$ value \\
\hline Cut-off
\end{tabular}

\begin{tabular}{lllll}
\hline Cut-off & 0.715 & 0.6575 & $4.98(1.64 ; 14.92)$ & 0.2713
\end{tabular}

Serologic I-FABP is a specific biomarker and is convenient to detect, which is primarily located in enterocytes of the small intestine and released into the circulation after NEC [5]. Other markers include C-reactive protein, an acute phase protein that becomes rapidly elevated with a series of infectious and inflammatory conditions. Several articles have proved CRP is a relatively sensitive (91\%) but nonspecific marker $(65 \%)$ for NEC early diagnosis [27, 28]. Like CRP, Trefoil factor 3 (TFF3) is also a sensitive ( $85 \%$ ) but nonspecific marker (59\%) for NEC [24]. Cytokines like IL-6 and IL-8 were thought to be reliable indicators; however, there were insufficient data available from individual studies assessing 


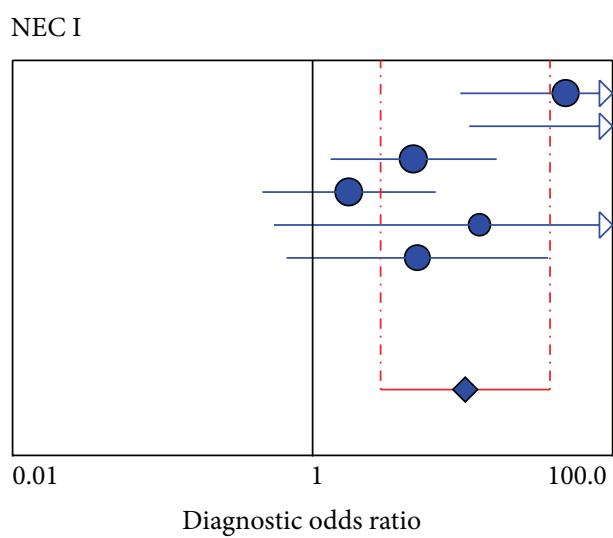

NEC II

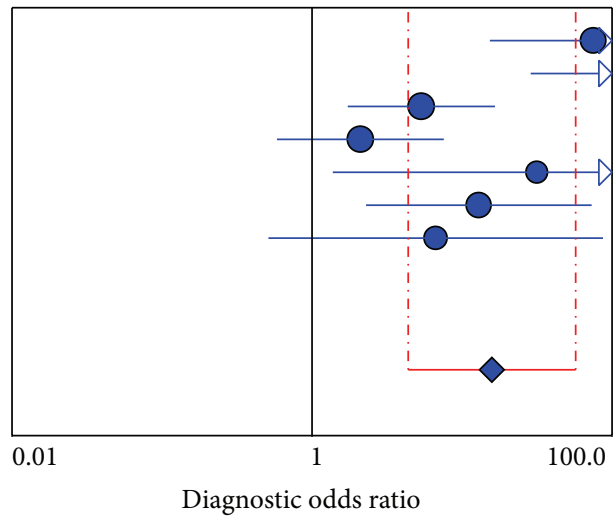

NEC III

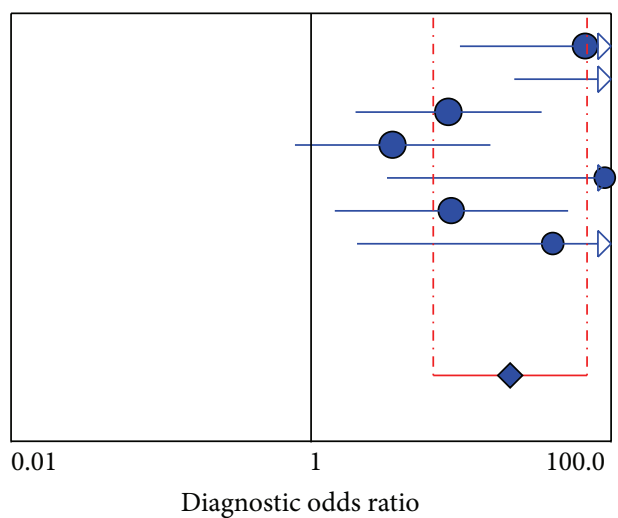

Aydemir et al. $2011 \quad 48.75(9.66-246.14)$

Benkoe et al. $2014 \quad 110.00(11.07-1,093.18)$

Edelson et al. $1999 \quad 4.71(1.32-16.87)$

Guan et al. $2014 \quad 1.75(0.46-6.62)$

Guthmann et al. $2002 \quad 13.00(0.55-306.21)$

Ren et al. $2013 \quad 5.00(0.67-37.26)$

Random-effects model

Pooled diagnostic odds ratio $=10.42$ (2.84 to 38.28 )

Cochran $-Q=15.79 ; \mathrm{df}=5(p=0.0075)$

Inconsistency $\left(I^{2}\right)=68.3 \%$

$\tau^{2}=1.7061$

Aydemir et al. 2011

Benkoe et al. 2014

Edelson et al. 1999

Guan et al. 2014

Guthmann et al. 2002

Ng et al. 2013

Ren et al. 2013

Diagnostic OR (95\% CI)

$75.00(15.34-366.65)$

$293.33(28.71-2,997.07)$

$5.36(1.73-16.60)$

$2.10(0.58-7.56)$

$31.57(1.37-725.23)$

$12.94(2.29-73.02)$

$6.67(0.51-86.93)$

Random-effects model

Pooled diagnostic odds ratio $=15.82$ (4.37 to 57.19)

Cochran $-Q=22.05 ; \mathrm{df}=6(p=0.0012)$

Inconsistency $\left(I^{2}\right)=72.8 \%$

$\tau^{2}=2.0419$

Aydemir et al. 2011

Benkoe et al. 2014

Edelson et al. 1999

Guan et al. 2014

Guthmann et al. 2002

$\mathrm{Ng}$ et al. 2013

Ren et al. 2013

Diagnostic OR (95\% CI)

$67.50(9.83-463.66)$

$396.00(22.61-6,935.94)$

$8.25(1.98-34.38)$

$3.50(0.78-15.69)$

$91.00(3.20-2,585.36)$

$8.63(1.44-51.72)$

$41.00(2.02-830.71)$

Random-effects model

Pooled diagnostic odds ratio $=21.26$ (6.53 to 69.21)

Cochran- $Q=13.58 ; \mathrm{df}=6(p=0.0346)$

Inconsistency $\left(I^{2}\right)=55.8 \%$

$\tau^{2}=1.3266$

FIGURE 4: Forest plot for DOR of I-FABP in NEC diagnosis. DOR: diagnostic odds ratios; NEC: necrotizing enterocolitis; I-FABP: intestinalfatty acid binding protein; CI: confidence interval.

cytokines in diagnosing NEC to present pooled estimates of diagnostic accuracy [27]. Reisinger et al. reported the combination of urinary Serum Amyloid A (SAA) with platelet count is an accurate detection method in diagnosing severe NEC, with higher sensitivity (94\%), specificity (83\%), and AUC area (0.95) [29]. Benkoe et al. reported a high diagnostic accuracy and clinically relevant value of fecal calprotectin $(\mathrm{AUC}=0.94)$ for diagnosing NEC, with a limitation in using the stool samples which cannot be obtained in some patients with NEC or non-NEC [20]. In addition, Aydemir et al. reported fecal calprotectin is a useful marker in differentiating severe NEC from early NEC with $76 \%$ sensitivity and $92 \%$ specificity [30].

An exploration of the source for heterogeneity rather than a summary computation was an important goal of this meta-analysis. Metaregression has proved that the cut-off value was the main factor, which may partially explain the heterogeneity because of the differences between studies. Hence, we expect to reduce the heterogeneity by including more studies for I-FABP test in the future. 


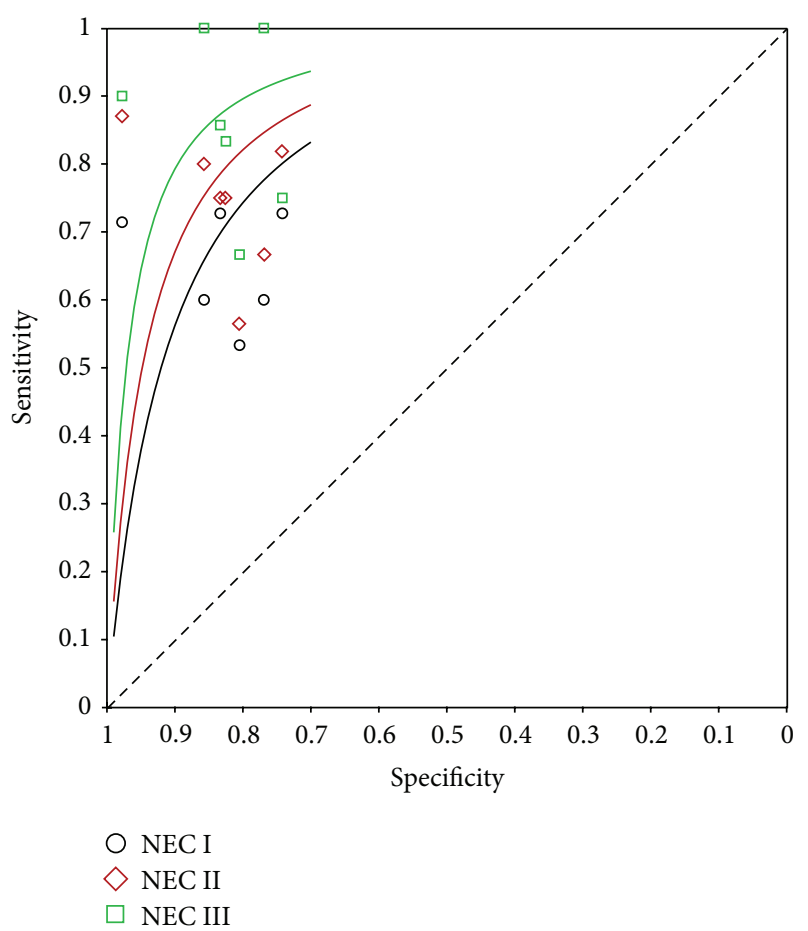

FIGURE 5: Summary receiver operating characteristic (SROC) curve of the I-FABP test for the diagnosis of NEC I, NEC II, and NEC III stages. I-FABP: intestinal-fatty acid binding protein; NEC: necrotizing enterocolitis; SROC: summary receiver operating characteristic.

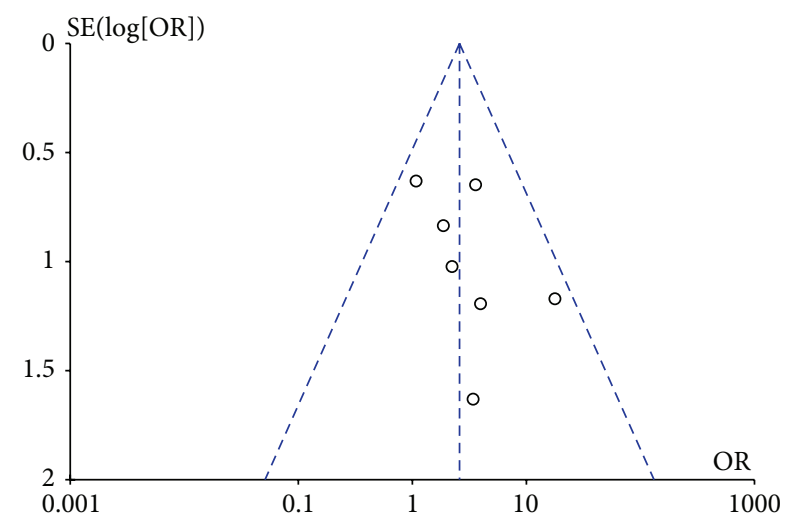

Figure 6: Funnel plot to estimate the publication bias of the metaanalysis.

The meta-analysis showed that serologic I-FABP was a helpful biomarker for early diagnosis of NEC for the premature neonates. Additionally, the study population in this review just included preterm neonates, and we supposed our findings may be also appropriate for early identifying full-term neonates with NEC, though the disease process in these two populations is markedly different [31]. However, few studies about full-term neonates could be searched to validate our supposition.

Although our results are valuable and promising, several limitations still existed in our study. First, the language barrier and limited studies might have led to some bias. In general, a large sample size can diminish any bias and make the conclusion more convincing. Second, some differences in the used ELISA's kits may also influence the results.

\section{Conclusion}

In summary, I-FABP is a valid serologic biomarker for early diagnosis of NEC for the premature neonates with a moderate accuracy; thus it may serve as a new auxiliary diagnosis method and decrease the omission diagnostic rate. To provide a more reliable diagnostic basis for clinical implement, further large scale and multicenter prospective studies are needed.

\section{Conflict of Interests}

The authors declare that they have no conflict of interests regarding the publication of this paper.

\section{Acknowledgments}

This work was supported by the Natural Science Foundation of Chongqing Grants (cstc2014jctjA10052) and the Foundation of Chongqing Medical University Institutions of China (lcyj2014-8).

\section{References}

[1] J. Neu and W. A. Walker, "Necrotizing enterocolitis," The New England Journal of Medicine, vol. 20, pp. 55-64, 2011.

[2] M. C. Walsh and R. M. Kliegman, "Necrotizing enterocolitis: treatment based on staging criteria," Pediatric Clinics of North America, vol. 33, no. 1, pp. 179-201, 1986.

[3] M. J. Bell, J. L. Ternberg, R. D. Feigin et al., "Neonatal necrotizing enterocolitis: therapeutic decisions based upon clinical staging," Annals of Surgery, vol. 187, no. 1, pp. 1-7, 1978.

[4] D. Dilli, Ş. Suna Oǧuz, R. Erol, H. Özkan-Ulu, H. DumanlI, and U. Dilmen, "Does abdominal sonography provide additional information over abdominal plain radiography for diagnosis of necrotizing enterocolitis in neonates?" Pediatric Surgery International, vol. 27, no. 3, pp. 321-327, 2011.

[5] M. M. A. L. Pelsers, W. T. Hermens, and J. F. C. Glatz, "Fatty acid-binding proteins as plasma markers of tissue injury," Clinica Chimica Acta, vol. 352, no. 1-2, pp. 15-35, 2005.

[6] G. Gollin, C. Marks, and W. H. Marks, "Intestinal fatty acid binding protein in serum and urine reflects early ischemic injury to the small bowel," Surgery, vol. 113, no. 5, pp. 545-551, 1993.

[7] J. M. Lieberman, J. Sacchettini, C. Marks, and W. H. Marks, "Human intestinal fatty acid binding protein: report of an assay with studies in normal volunteers and intestinal ischemia," Surgery, vol. 121, no. 3, pp. 335-342, 1997.

[8] P. Whiting, A. W. S. Rutjes, J. B. Reitsma, P. M. M. Bossuyt, and J. Kleijnen, "The development of QUADAS: a tool for the quality assessment of studies of diagnostic accuracy included in systematic reviews," BMC Medical Research Methodology, vol. 3, article 25, 2003.

[9] J. Zamora, V. Abraira, A. Muriel, K. Khan, and A. Coomarasamy, "Meta-DiSc: a software for meta-analysis of test accuracy data," BMC Medical Research Methodology, vol. 6, article 31, 2006. 
[10] W. L. Deville, F. Buntinx, L. M. Bouter et al., "Conducting systematic reviews of diagnostic studies: didactic guidelines," BMC Medical Research Methodology, vol. 2, article 9, pp. 23-29, 2002.

[11] L. Irwig, A. N. A. Tosteson, C. Gatsonis et al., "Guidelines for meta-analyses evaluating diagnostic tests," Annals of Internal Medicine, vol. 120, no. 8, pp. 667-676, 1994.

[12] E. C. Vamvakas, "Meta-analysis of studies of the diagnostic accuracy of laboratory tests: a review of the concepts and methods," Archives of Pathology \& Laboratory Medicine, vol.122, pp. 85-86, 1998.

[13] J. B. Reitsma, A. S. Glas, A. W. S. Rutjes, R. J. P. M. Scholten, P. M. Bossuyt, and A. H. Zwinderman, "Bivariate analysis of sensitivity and specificity produces informative summary measures in diagnostic reviews," Journal of Clinical Epidemiology, vol. 58, no. 10, pp. 982-990, 2005.

[14] T. J. Cleophas and A. H. Zwinderman, "Meta-analyses of diagnostic studies," Clinical Chemistry and Laboratory Medicine, vol. 47, no. 11, pp. 1351-1354, 2009.

[15] L. R. Arends, T. H. Hamza, J. C. van Houwelingen, M. H. Heijenbrok-Kal, M. G. M. Hunink, and T. Stijnen, "Bivariate random effects meta-analysis of ROC curves," Medical Decision Making, vol. 28, no. 5, pp. 621-638, 2008.

[16] C. M. Jones and T. Athanasiou, "Summary receiver operating characteristic curve analysis techniques in the evaluation of diagnostic tests," The Annals of Thoracic Surgery, vol. 79, no. 1, pp. 16-20, 2005.

[17] J. G. Lijmer, P. M. M. Bossuyt, and S. H. Heisterkamp, "Exploring sources of heterogeneity in systematic reviews of diagnostic tests," Statistics in Medicine, vol. 21, no. 11, pp. 1525-1537, 2002.

[18] J. J. Deeks, P. Macaskill, and L. Irwig, “The performance of tests of publication bias and other sample size effects in systematic reviews of diagnostic test accuracy was assessed," Journal of Clinical Epidemiology, vol. 58, no. 9, pp. 882-893, 2005.

[19] C. Aydemir, D. Dilli, S. S. Oguz et al., "Serum intestinal fatty acid binding protein level for early diagnosis and prediction of severity of necrotizing enterocolitis," Early Human Development, vol. 87, no. 10, pp. 659-661, 2011.

[20] T. M. Benkoe, T. P. Mechtler, M. Weninger, M. Pones, W. Rebhandl, and D. C. Kasper, "Serum levels of interleukin8 and gut-associated biomarkers in diagnosing necrotizing enterocolitis in preterm infants," Journal of Pediatric Surgery, vol. 49, no. 10, pp. 1446-1451, 2014.

[21] M. B. Edelson, R. E. Sonnino, C. E. Bagwell, J. M. Lieberman, W. H. Marks, and H. J. Rozycki, "Plasma intestinal fatty acid binding protein in neonates with necrotizing entercolitis: a pilot study," Journal of Pediatric Surgery, vol. 34, no. 10, pp. 1453-1457, 1999.

[22] H. S. Guan, A. P. Yan, and Y. H. Liu, "Significance of plasma intestinal fatty acid binding protein level in neonatal necrotizing enterocolitis," Chinese Journal of Neonatology, vol. 3, pp. 29-30, 2014.

[23] F. Guthmann, T. Börchers, C. Wolfrum, T. Wustrack, S. Bartholomäus, and F. Spener, "Plasma concentration of intestinal- and liver-FABP in neonates suffering from necrotizing enterocolitis and in healthy preterm neonates," Molecular and Cellular Biochemistry, vol. 239, no. 1-2, pp. 227-234, 2002.

[24] E. W. Y. Ng, T. C. W. Poon, H. S. Lam et al., "Gut-associated biomarkers L-FABP, I-FABP, and TFF3 and LIT score for diagnosis of surgical necrotizing enterocolitis in preterm infants," Annals of Surgery, vol. 258, no. 6, pp. 1111-1118, 2013.
[25] X. Y. Ren, C. Li, and Y. X. Cai, "Serum intestinal fatty acid binding protein in the earlydiagnosis of NEC," Chinese Medical Journal, 2013.

[26] C. R. Huang-Fu, P. Li, and Y. F. Tian, "Clinical significance of serum intestinal fatty acid-binding protein in infants with necrotizing enterocolitis," Zhongguo Dang Dai Er Ke Za Zhi, vol. 16, pp. 25-28, 2014.

[27] N. Evennett, N. Alexander, M. Petrov, A. Pierro, and S. Eaton, "A systematic review of serologic tests in the diagnosis of necrotizing enterocolitis," Journal of Pediatric Surgery, vol. 44, no. 11, pp. 2192-2201, 2009.

[28] C. Young, R. Sharma, M. Handfield, V. Mai, and J. Neu, "Biomarkers for infants at risk for necrotizing enterocolitis: clues to prevention?” Pediatric Research, vol. 65, no. 5, pp. 91-97, 2009.

[29] K. W. Reisinger, B. W. Kramer, D. C. van der Zee et al., "Noninvasive serum amyloid A (SAA) measurement and plasma platelets for accurate prediction of surgical intervention in severe necrotizing enterocolitis (NEC)," PLoS ONE, vol. 9, no. 3, Article ID e90834, 2014.

[30] O. Aydemir, C. Aydemir, Y. U. Sarikabadayi et al., "Fecal calprotectin levels are increased in infants with necrotizing enterocolitis," The Journal of Maternal-Fetal \& Neonatal Medicine, vol. 25, no. 11, pp. 2237-2241, 2012.

[31] E. H. Raboel, "Necrotizing enterocolitis in full-term neonates: is it aganglionosis?" European Journal of Pediatric Surgery, vol. 19, no. 2, pp. 101-104, 2009. 


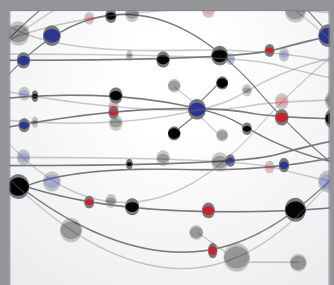

The Scientific World Journal
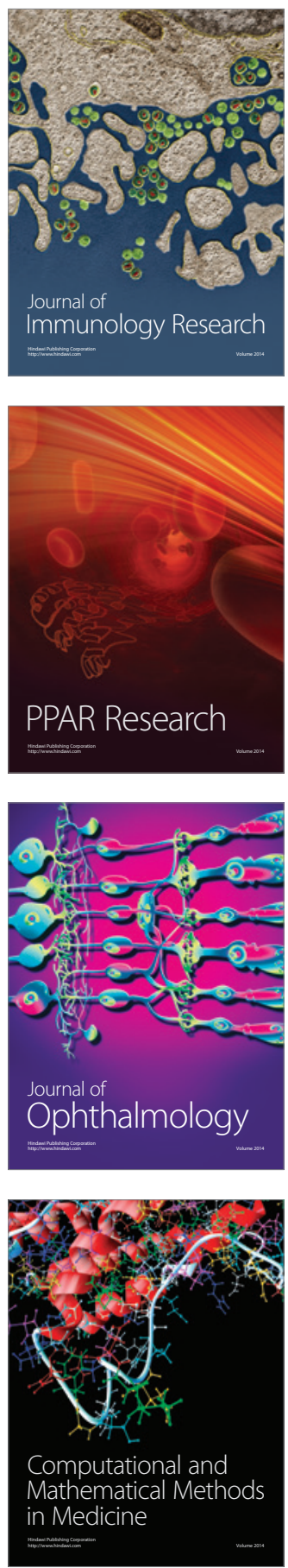

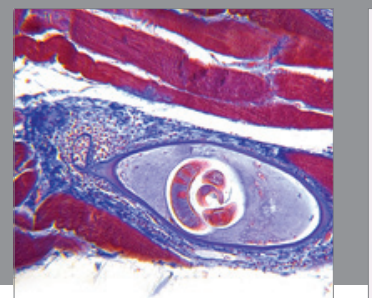

Gastroenterology

Research and Practice
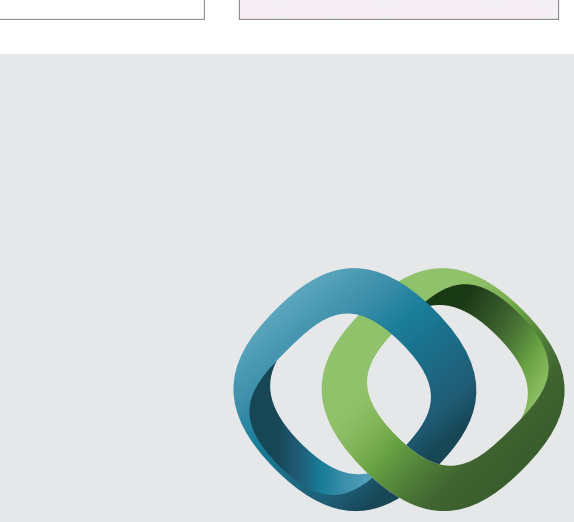

\section{Hindawi}

Submit your manuscripts at

http://www.hindawi.com
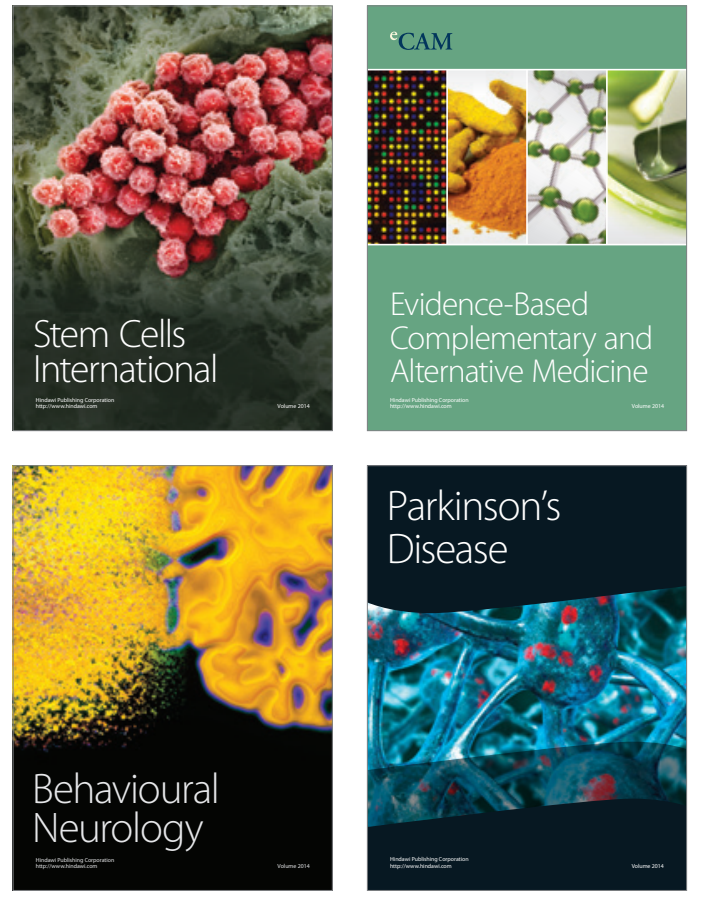
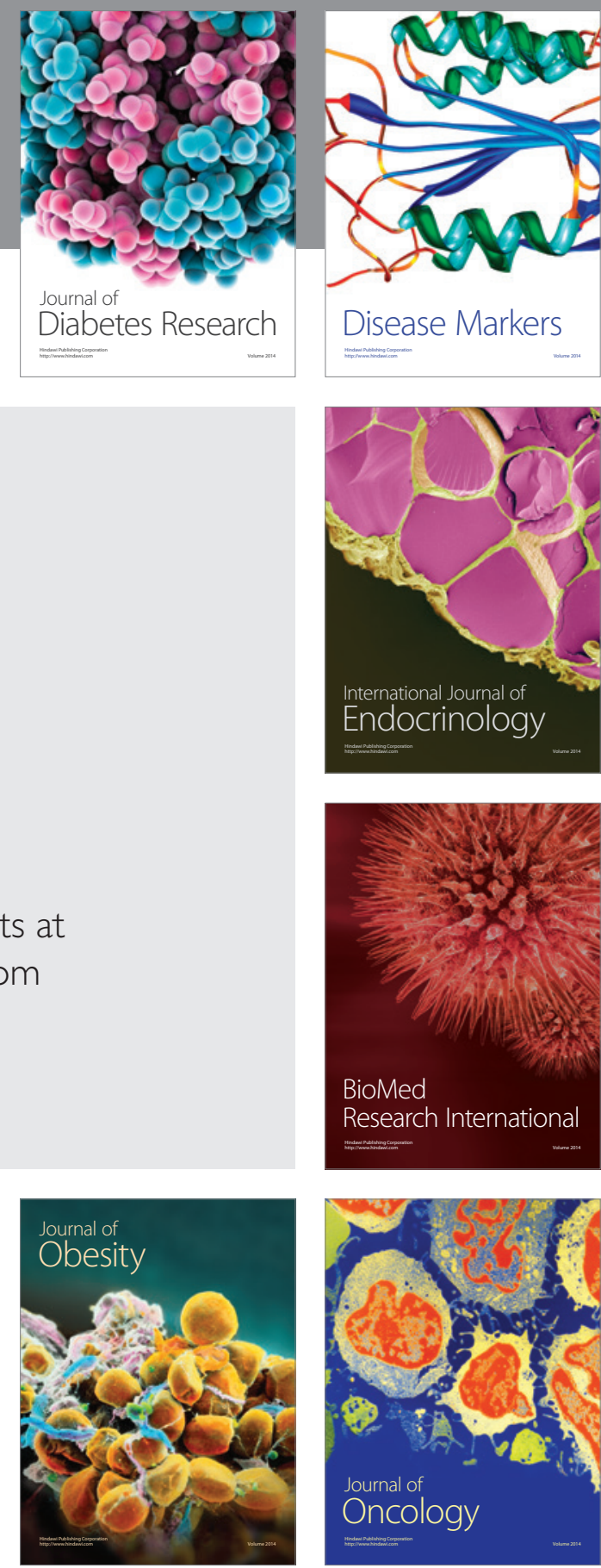

Disease Markers
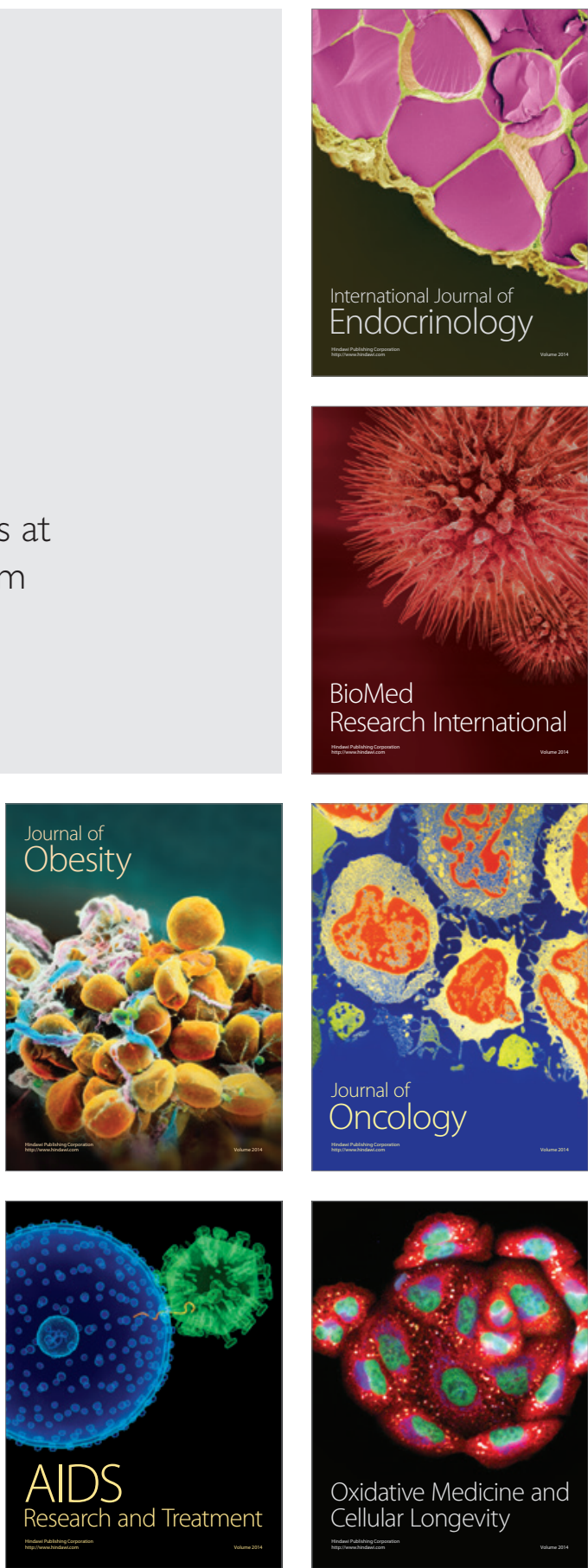